\title{
Periurethral Region
}

National Cancer Institute

\section{Source}

National Cancer Institute. Periurethral Region. NCI Thesaurus. Code C111287.

The tissue surrounding the urethra. 